The methodological pipeline described here is adequate to characterize in-depth mutant spectra of $\mathrm{HCV}$ populations, and it provides a tool to understand HCV diversification and treatment failures. The pipeline can be periodically extended in the event of HCV diversification into new genotypes or subtypes, and provides a framework applicable to other RNA viral pathogens, with potential to couple detection of drug-resistant mutations with treatment planning.

\subsection{7}

doi: 10.15789/2220-7619-2018-4-7.17

\section{THE SIGNIFICANCE OF THE HIV RESISTANCE ANALYSIS IN ANTIRETROVIRAL THERAPY}

\section{D.E. Valutite, Yu.V. Ostankova}

\section{St. Petersburg Pasteur Institute, St. Petersburg, Russia}

The widespread use of antiretroviral therapy, the development of new drugs and treatment regimens are inherently associated with the emergence of HIV resistance. Systematic laboratory monitoring of patients with ineffectiveness of antiretroviral therapy is necessary.

Objective was to assess the significance of the resistance analysis in the current algorithms for control HIV infection in individuals.

Blood plasma samples of 66 patients from different regions of the North West Federal District were used in the work. All patients were referred for testing the drug resistance of the virus due to the virological ineffectiveness of antiretroviral therapy. The nucleotide sequences obtained with the use of a set of reagents for the detection of mutations of drug resistance to antiretroviral drugs AmpliSens ${ }^{\circledR}$ HIV-Resist-Seq.

For this group of patients two main scheme of therapies were used: the first one included two drugs from the Nucleoside Reverse Transcriptase Inhibitors group in combination with one drug from the Non-Nucleoside Reverse Transcriptase Inhibitors group and the second one from the Protease Inhibitors group in combination with two drugs from the Nucleoside Reverse Transcriptase Inhibitors group. The analysis of nucleotide sequences in the protease gene showed that most commonly there are mutations that cause drug resistance to groups of nucleoside and non-nucleoside reverse transcriptase inhibitors. In 45 patients $(60 \%)$ a mutation in the position of $\mathrm{M} 184 \mathrm{~V}$ was detected, which is responsible for the presence of a high level of resistance to lamivudine. In $34(52 \%)$ cases, a mutation was found in the K65R position, responsible for the cause of resistance to abacavir and didanosine. In $12(18 \%)$ patients, a combination of mutations M184V + L74V, also resulting in resistance to didanosine and abacavir, was detected. This indicates that when the therapy is changed, the percentage of the most frequent mutations increases, along with the appearance of new ones, which indicates the need for an analysis of the resistance of HIV to antiretroviral therapy not only in cases of virological inefficiency of therapy, but also during the change of therapy and similarly, naive patients should be analyzed for existing mutations to optimize treatment.

\section{FORECASTING OF INCIDENCE OF HAV WITH USE OF THE SCHEDULED PLAN OF WALD}

V.M. Volkova ${ }^{1}$, O.A. Burgasova ${ }^{2}$, V.B. Postupailo ${ }^{3}$, L.V. Sayapina ${ }^{3}$ ${ }^{1}$ Russian Medical Academy of Continuous Professional Education, Moscow, Russia; ${ }^{2}$ Peoples' Friendship University of Russia (RUDN University), Moscow, Russia; ${ }^{3}$ Scientific Centre of Medical Products, Moscow, Russia

The incidence of viral hepatitis A (HAV) has high epidemiological significance. According to The Federal service for supervision of consumer rights protection and human welfare in Russian Federation during 20102016. 44666 cases of HAV among adult population, and the children's population 21308 respectively are registered.

For the purpose of assessment of an epidemiological situation on HAV the analysis of official statistical data on incidence of HAV is carried out to the Moscow in 2016 and forecasting of an epidemic situation for 2017. The forecast of number of cases was carried out with use of linear approximation and the developed method of forecasting of incidence of HAV with use the plan - Wald's graphics.

It is established by the results of the analysis of the dynamics of the incidence of HAV in Moscow, using the method of linear approximation in 2017, the incidence of HAV in adults and children was 240 and 115 cases These statistical indicators characterize the epidemiological situation as unfavorable.

According to the results of the statistical analysis, the threshold level of the incidence of HAV in Moscow among children for the period 2010-2016 analyzed. 11 cases were made, which is defined as an incremental total, with a minimum monthly prognostic level of 7 cases, a maximum of 17. The monthly increase in the number of diseases in the dynamics of the analyzed year was 0.9 cases. The forecast of the maximum number of diseases of the HAV in 2017 exceeds the threshold level of morbidity and indicates a possible worsening of the epidemiological situation in Moscow. The forecast of the total minimum and maximum incidence rate in 2017 will be 84 and 204 cases respectively.

Based on the results of the analysis of the incidence of HAV for the period from 2010 to 2016, using the Wald schedule it was shown that the epidemic situation in Moscow among the adult population and among the children under 17 is estimated as unstable. The method of analysis and forecasting using the Wald schedule allows to predict the total minimum and maximum levels of the incidence of HAV, which is of great importance for practical public health and makes it possible to adjust planned preventive and antiepidemic measures.

\section{RISK FACTORS OF PERINATAL TRANSMISSION OF HEPATITIS C VIRUS}

\section{T.V. Znovets, E.I. Baranovskaya, S.V. Zhavoronok}

Belarusian State Medical University, Minsk, Republic of Belarus

Worldwide, the virus of hepatitis C (HCV) infected $2-3 \%$ of the population (more than 185 million people). The proportion of pregnant women with hepatitis $\mathrm{C}(\mathrm{HC})$ is $1-2.4 \%$. In previous studies, it was reported that perinatal transmission of $\mathrm{HCV}$ is due to the level of viral load of HCV, HIV co-infection, increased activity of alanine aminotransferase, duration of anhydrous period. The use of amniocentesis and internal monitoring of the fetus may increase the risk of perinatal transmission of HCV. There is no evidence of a reduction in the risk of HCV transmission from the mother to the baby in cesarean section and the rejection of breastfeeding.

The aim of the study was to determine the risk factors for perinatal transmission of the hepatitis $\mathrm{C}$ virus.

To diagnose perinatal transmission of HCV, 140 children born to women with HS were examined. The diagnosis of congenital HS was established when HCV RNA was detected in the blood plasma of a child older than 2 months twice with an interval of at least 3 months or anti-HCV in a child over 18 months old using commercial reagent kits. The ELISA method was used to detect antibodies to HCV ("ELISAHCV-Ab", The Republican Scientific and Practical Center for Epidemiology and Microbiology, Belarus, Monolisa HCV 